\title{
Delineamento em blocos casualizados nas pesquisas em ciências agrárias: Uma revisão integrativa de literatura
}

\author{
Randomized block design in agricultural Science research: An integrative literature review \\ Diseño de bloques aleatórios en la investigación en ciencias agrícolas: Uma revisión integradora de \\ la literatura
}

Recebido: 27/06/2021 | Revisado: 04/07/2021 | Aceito: 07/07/2021 | Publicado: 17/07/2021

\author{
Wendel dos Santos Moraes \\ ORCID: https://orcid.org/0000-0002-6791-4478 \\ Universidade Federal do Tocantins, Brasil \\ E-mail: wmorais26@gmail.com \\ Octávio Henrique Bernardes Barros \\ ORCID: https://orcid.org/0000-0002-3425-8152 \\ Universidade Federal do Tocantins, Brasil \\ E-mail: octaviobbarros@gmail.com \\ Hellen Cristina de Freitas \\ ORCID: https://orcid.org/0000-0003-1760-0152 \\ Universidade Federal do Tocantins, Brasil \\ E-mail: enghellencris@gmail.com \\ Ana Clara Carneiro \\ ORCID: https://orcid.org/0000-0002-2906-5380 \\ Universidade Federal do Tocantins, Brasil \\ E-mail: anaclarafloresteira@gmail.com \\ Gabriella Rayssa Antunes da Silva Oliveira \\ ORCID: https://orcid.org/0000-0002-4116-6635 \\ Universidade Federal do Tocantins, Brasil \\ E-mail: gabriellarayssa.antunes@gmail.com \\ Daniela de Oliveira Luciano \\ ORCID: https://orcid.org/0000-0003-0768-0339 \\ Universidade Federal do Tocantins, Brasil \\ E-mail: daniela.luciano@mail.uft.edu.br \\ Augustus Caeser Franke Portella \\ ORCID: https://orcid.org/0000-0001-6988-0622 \\ Universidade Federal do Tocantins, Brasil \\ E-mail: portella@mail.uft.edu.br
}

\begin{abstract}
Resumo
No processo de desenvolvimento das pesquisas em ciências agrárias existem diferentes fatores que influem no manejo das culturas. O delineamento em blocos casualizados (DBC) é uma técnica utilizada para analisar experimentos com um conjunto de fatores não análogas em uma determinada localidade e seu impacto no experimento. Os tratamentos são ao acaso sobre as unidades experimentais dentro de cada bloco, com isso, esse método é usado para verificar se há interferência entre os tratamentos e entre os blocos. Desta forma, o presente trabalho teve como objetivo realizar uma revisão integrativa de literatura sobre as aplicações do DBC nas pesquisas da área de ciências agrárias. A metodologia aplicada foi a de revisão integrativa de literatura. A escolha dos artigos para composição da amostra se deu por intermédio de periódicos publicado de 2010 a 2020, das bases de dados Scielo, Google Scholar, e Science Direct. E posteriormente, os achados foram separados nos ramos da área de ciências agrárias conforme a classificação da CAPES. Obteve-se 41 estudos que atenderam aos critérios de inclusão estabelecidos. Dos trabalhos adquiridos 22 $(53,6 \%)$ da base de dados da Scielo, 19 (46,3\%) do Google Scholar, e 1 (2,4\%) da Science Direct. 23 (56,0\%) se enquadram na área de Agronomia, e 18 (43,9\%) a área de Recursos florestais e engenharia florestal. Os resultados deste estudo demostram que o DBC tem contribuído para o avanço dos diferentes campos das áreas da Ciências Agrárias, possibilitando uma melhor tabulação dos dados adquiridos, e facilitando na análise das unidades experimentais.
\end{abstract}

Palavras-chave: Fatores; Análise; Unidades experimentais.

\begin{abstract}
In the development process of research in agricultural sciences, there are different factors that influence crop management. The randomized block design (DBC) is a technique used to analyze experiments with a set of nonanalogous factors in a given location and their impact on the experiment. Treatments are randomized over the
\end{abstract}


experimental units within each block, so this method is used to check for interference between treatments and between blocks. Thus, the present work aimed to carry out an integrative literature review on the applications of the DBC in research in the field of agricultural sciences. The methodology applied was an integrative literature review. The choice of articles to compose the sample was made through journals published from 2010 to 2020, from the Scielo, Google Scholar, and Science Direct databases. And later, the findings were separated into branches of the agricultural sciences area according to the CAPES classification. 41 studies that met the established inclusion criteria were obtained. Of the works acquired, $22(53.6 \%)$ from the Scielo database, 19 (46.3\%) from Google Scholar, and 1 (2.4\%) from Science Direct. $23(56.0 \%)$ fall into the area of Agronomy, and 18 (43.9\%) fall into the area of Forest Resources and Forest Engineering. The results of this study show that the DBC has contributed to the advancement of different fields in the areas of Agrarian Sciences, enabling a better tabulation of acquired data, and facilitating the analysis of experimental units.

Keywords: Factors; Analyze; Experimental units.

\section{Resumen}

En el proceso de desarrollo de la investigación en ciencias agrícolas, existen diferentes factores que influyen en el manejo de los cultivos. El diseño de bloques aleatorios (DBC) es una técnica utilizada para analizar experimentos con un conjunto de factores no análogos en una ubicación determinada y su impacto en el experimento. Los tratamientos se asignan al azar en las unidades experimentales dentro de cada bloque, por lo que este método se utiliza para verificar la interferencia entre tratamientos y entre bloques. Así, el presente trabajo tuvo como objetivo realizar una revisión bibliográfica integradora sobre las aplicaciones del DBC en la investigación en el campo de las ciencias agrícolas. La metodología aplicada fue una revisión integradora de la literatura. La elección de los artículos para componer la muestra se realizó a través de revistas publicadas de 2010 a 2020, de las bases de datos Scielo, Google Scholar y Science Direct. Y posteriormente, los hallazgos se separaron en ramas del área de ciencias agrícolas según la clasificación CAPES. Se obtuvieron 41 estudios que cumplieron con los criterios de inclusión establecidos. De los trabajos adquiridos, $22(53,6 \%)$ de la base de datos Scielo, 19 (46,3\%) de Google Scholar y 1 (2,4\%) de Science Direct. $23(56,0 \%)$ corresponden al área de Agronomía y 18 (43,9\%) al área de Recursos Forestales e Ingeniería Forestal. Los resultados de este estudio muestran que el DBC ha contribuido al avance de diferentes campos en las áreas de las Ciencias Agrarias, permitiendo una mejor tabulación de los datos adquiridos y facilitando el análisis de unidades experimentales.

Palabras clave: Factores; Analizar; Unidades experimentales.

\section{Introdução}

O delineamento em blocos ao acaso ou casualizados (DBC) é uma técnica da estatística experimental que considera os três princípios básicos da experimentação que são: repetição, casualização, e controle local (Pimentel-Gomes, 2009). Esse tipo de delineamento é tido como o mais usado em detrimento dos outros tipos, geralmente é utilizado para experimentos implantados a campo, tendo em vista que o conjunto de fatores em uma determinada localidade não são análogas e isso pode ter impacto significativo no experimento (Costa, 2003).

Portanto, os blocos são um conjunto de unidades experimentais (UE) homogêneas, podendo ser, parcelas com a mesma cota (curva de nível), árvores alturas iguais, espécie e diâmetro; animais oriundos de mesma filiação; cortes de madeira de uma mesma árvore (Alves, 2019). Cada bloco recebe um tratamento (repetição) e, assim, o número de unidade experimental por bloco é igual ao número de tratamentos (Kronka, 2018). Os tratamentos são ao acaso sobre as unidades experimentais dentro de cada bloco, com isso, esse método é usado para verificar se há interferência entre os tratamentos e entre os blocos (Cargnelutti, 2009).

No processo de desenvolvimento das pesquisas em ciências agrárias é observado diferentes fatores que influem no manejo das culturas e nas características do produto final. O pesquisador faz uso da experimentação para verificar quais as variáveis de maior importância na produção, assim comprovando ou corroborando suas hipóteses formuladas (Padovani, 2014). Definir qual o delineamento experimental é importante, pois ele irá conduzir o perfil do experimento. Deste modo, o presente trabalho teve como objetivo realizar uma revisão integrativa de literatura sobre as aplicações do delineamento experimental em blocos casualizados nas pesquisas da área de ciências agrárias. 


\section{Metodologia}

Os procedimentos metodológicos aplicados no desenvolvimento deste trabalho foram com base no Manual de Revisão Bibliográfica Sistemática Integrativa (2014). A revisão integrativa de literatura concerne no compilado de resultados obtidos em pesquisas científicas que auxiliam na fundamentação sistemática de uma determinada temática (Souza; Silva; Carvalho, 2010).

O levantamento dos achados selecionados foi realizado com as palavras chave: Delineamento em blocos casualizados, Ciências agrárias, Análise de dados, Repetição, Randomização. Considerou-se também o enquadramento das publicações na grande área de ciências agrárias conforme a classificação da Coordenação de Aperfeiçoamento de Pessoal de Nível Superior (CAPES) das áreas de Agronomia, Recursos florestais e engenharia florestal, Engenharia agrícola e suas respectivas subáreas (Quadro 1).

Quadro 1 - Áreas do conhecimento nas ciências agrárias.

\begin{tabular}{|l|l|}
\hline Áreas & Subáreas \\
\hline \multirow{5}{*}{ Agronomia } & Ciência do Solo \\
& $\begin{array}{l}\text { Fitossanidade } \\
\text { Fitotecnia } \\
\text { Floricultura, Parques e Jardins } \\
\text { Agrometeorologia } \\
\text { Extensão Rural }\end{array}$ \\
\hline Recursos florestais e engenharia florestal & $\begin{array}{l}\text { Silvicultura } \\
\text { Manejo Florestal } \\
\text { Técnicas e Operações Florestais } \\
\text { Tecnologia e Utilização de Produtos Florestais } \\
\text { Conservação da Natureza } \\
\text { Energia de Biomassa Florestal }\end{array}$ \\
\hline \multirow{5}{*}{ Engenharia agrícola } & $\begin{array}{l}\text { Máquinas e Implementos Agrícolas } \\
\text { Engenharia de Água e Solo } \\
\text { Engenharia de Processamento de Produtos Agrícolas } \\
\text { Construções Rurais e Ambiência } \\
\text { Energização Rural }\end{array}$ \\
\hline
\end{tabular}

Fonte: CAPES (2021).

Este estudo teve como questão norteadora: Qual a colaboração do delineamento em blocos casualizados para a eficiência no desenvolvimento dos experimentos na área de ciências agrárias?

A escolha dos artigos para composição da amostra se deu por intermédio de consulta nas bases de dados Scielo, e Google Scholar. Como critério de inclusão foram considerados artigos completos, em periódicos publicados de 2010 até 2020, em português e inglês. Os artigos repetidos em duas bases de dados foram incluídos na base de dado que apresentou a maior quantidade de artigos. Após o levantamento dos estudos foi realizada uma leitura dos títulos e resumos com intuito de verificar a concordância em relação à questão norteadora.

\section{Resultados}

Nesta revisão integrativa foram incluídos 41 estudos que atenderam aos critérios de inclusão estabelecidos. Dos 41 trabalhos foram adquiridos $22(53,6 \%)$ da base de dados da Scielo, 19 (46,3\%) do Google Scholar, e 1 (2,4\%) da Science Direct. Percebeu-se em relação a distribuição dos achados no recorte temporal dos últimos 10 anos que dois (4,8\%) são do ano 
de 2010; sete (17\%) são do ano de 2011; dez (24,3\%) são artigos publicados em 2012; seis (14,6\%) do ano de 2013; três $(7,3 \%)$ artigos em 2014; quatro (9,7\%) no ano de 2015; dois (4,8\%) do ano de 2016; quatro (9,7\%) artigos do ano de 2017; e três $(7,3 \%)$ do ano de 2020. Nos anos de 2018 e 2019 não há pesquisas que se enquadraram nos critérios estabelecidos.

No Quadro 2 nota-se a distribuição dos estudos selecionados para esse estudo apresentando autor, ano, título, e periódico publicado.

Quadro 2 - Composição dos estudos segundo autor, ano, título, e periódico.

\begin{tabular}{|c|c|c|}
\hline Autores e Ano da Publicação & Título & Periódico \\
\hline $\begin{array}{l}\text { Itamar Rosa Teixeira, Gisele Carneiro da Silva, João Paulo Ribeiro de } \\
\text { Oliveira, Alessandro Guerra da Silva, Adilson Pelá, } 2010 .\end{array}$ & $\begin{array}{l}\text { Desempenho agronômico e qualidade de } \\
\text { sementes de cultivares de feijão-caupi na } \\
\text { região do cerrado. }\end{array}$ & Revista Ciência Agronômica \\
\hline $\begin{array}{l}\text { Pedro Paulo Gomes de Oliveira, Hugo Armando Barúa Acosta, } \\
\text { Luciano de Souza Maria e Marco Antônio Camillo de Carvalho, } 2010 .\end{array}$ & $\begin{array}{l}\text { Influência da desrama artificial sobre o } \\
\text { crescimento da teca (Tectona grandis) no } \\
\text { Estado de Rondônia. }\end{array}$ & $\begin{array}{l}\text { Embrapa Rondônia. Circular } \\
\text { técnica. }\end{array}$ \\
\hline $\begin{array}{l}\text { Eduardo Fávero Caires, Evandro Henrique Gonçalves Maschietto, } \\
\text { Fernando José Garbuio, Susana Churka, Hélio Antonio Wood Joris, } \\
2011 .\end{array}$ & $\begin{array}{l}\text { Surface application of gypsum in low } \\
\text { acidic Oxisol under no-till cropping } \\
\text { system. }\end{array}$ & Scientia Agricola, Piracicaba \\
\hline $\begin{array}{l}\text { Márcia Maria Dias, Nilton Nagib Jorge Chalfun, Silvério José Coelho } \\
\text { e Verônica Andrade dos Santos, } 2011 .\end{array}$ & $\begin{array}{l}\text { Meios de diluição e concentrações de } \\
\text { ácido indolbutírico no enraizamento de } \\
\text { estacas de cerejeira ornamental. }\end{array}$ & $\begin{array}{l}\text { Tecnologia \& Ciência. } \\
\text { Agropecuária }\end{array}$ \\
\hline $\begin{array}{l}\text { Pedro Dantas Fernandes, Marcos Eric Barbosa Brito, Hans Raj Gheyi, } \\
\text { Walter dos Santos Soares Filho, Alberto Soares de Melo, Paulo Torres } \\
\text { Carneiro, 2011. }\end{array}$ & $\begin{array}{l}\text { Crescimento de híbridos e variedades } \\
\text { porta-enxerto de citros sob salinidade. }\end{array}$ & Acta Scientiarum. Agronomy \\
\hline $\begin{array}{l}\text { Damiana Cleuma de Medeiros, José Francismar de Medeiros, } \\
\text { Pollyana Mona Soares Dias, Murillo Anderson Gonçalves Barbosa, } \\
\text { Keivianne da Silva Lima, } 2011 .\end{array}$ & $\begin{array}{l}\text { Produção de melão Pele de Sapo híbrido } \\
\text { 'Medellín' cultivado com diferentes } \\
\text { salinidades da água. }\end{array}$ & Revista Ciência Agronômica. \\
\hline $\begin{array}{l}\text { José George Ferreira Medeiros, Altamiro Oliveira Malta, Nivânia } \\
\text { Pereira Costa, Raunira Costa Araújo, Elton Lúcio Araújo, } 2011 .\end{array}$ & $\begin{array}{l}\text { Substâncias Atrativas no Monitoramento } \\
\text { de Moscas-dasfrutas em Goiabeiras e } \\
\text { Mangueiras no Município de } \\
\text { Bananeiras-PB. }\end{array}$ & $\begin{array}{l}\text { Revista Verde de } \\
\text { Agroecologia e } \\
\text { Desenvolvimento Sustentável }\end{array}$ \\
\hline $\begin{array}{l}\text { Itacir Eloi Sandini, Anibal de Moraes, Adelino Pelissari, Mikael } \\
\text { Neumann, Margarete Kimie Falbo, Jaqueline Huzar Novakowiski, } \\
2011 .\end{array}$ & $\begin{array}{l}\text { Efeito residual do nitrogênio na cultura } \\
\text { do milho no sistema de produção } \\
\text { integração lavoura-pecuária. }\end{array}$ & Ciência Rural \\
\hline $\begin{array}{l}\text { Alexandre Marques da Silva, Mario Luiz Teixeira de Moraes, Salatiér } \\
\text { Buzetti, } 2011 .\end{array}$ & $\begin{array}{l}\text { Propriedades químicas de solo sob } \\
\text { reflorestamento ciliar após } 20 \text { anos de } \\
\text { plantio em área de cerrado. }\end{array}$ & Revista Árvore \\
\hline $\begin{array}{l}\text { Jarina Idália Avelino, Jailma Suerda Silva de Lima, Maria Clarete } \\
\text { Cardoso Ribeiro, Aridênia Peixoto Chaves, Gardênia Silvana de } \\
\text { Oliveira Rodrigues, } 2012 .\end{array}$ & $\begin{array}{l}\text { Métodos de quebra de dormência em } \\
\text { sementes de jucá (Caesalpinia ferrea } \\
\text { Mart. ex Tul. var. Ferrea) }\end{array}$ & $\begin{array}{l}\text { Revista Verde de } \\
\text { Agroecologia e } \\
\text { Desenvolvimento Sustentável }\end{array}$ \\
\hline $\begin{array}{l}\text { Tiago Reis Dutra, Paulo Henrique Grazziotti, Reynaldo Campos } \\
\text { Santana, Marília Dutra Massad, } 2012 .\end{array}$ & $\begin{array}{l}\text { Desenvolvimento inicial de mudas de } \\
\text { copaíba sob diferentes níveis de } \\
\text { sombreamento e substratos. }\end{array}$ & Revista Ciência Agronômica \\
\hline $\begin{array}{l}\text { Marlo P Maciel, Tales M Soares, Hans R Gheyi, Emerson P. L } \\
\text { Rezende, Greice X. S Oliveira, } 2012 .\end{array}$ & $\begin{array}{l}\text { Produção de girassol ornamental com } \\
\text { uso de águas salobras em sistema } \\
\text { hidropônico NFT. }\end{array}$ & $\begin{array}{l}\text { Revista Brasileira de } \\
\text { Engenharia Agrícola e } \\
\text { Ambiental. }\end{array}$ \\
\hline $\begin{array}{l}\text { Ademir Kleber Morbeck de Oliveira, Sônia Cristina Juliano de } \\
\text { Gualtieri Andrade e Perez, } 2012 .\end{array}$ & $\begin{array}{l}\text { Crescimento inicial de Tabebuia aurea } \\
\text { sob três intensidades luminosas. }\end{array}$ & Ciência Florestal \\
\hline $\begin{array}{l}\text { Delfran Batista dos Santos, Paulo Afonso Ferreira, Flavio Gonçalves } \\
\text { de Oliveira, Rafael Oliveira Batista, Alan Carlos Costa, Marco } \\
\text { Antonio Oliva Cano, } 2012 .\end{array}$ & $\begin{array}{l}\text { Produção e parâmetros fisiológicos do } \\
\text { amendoim em função do estresse salino. }\end{array}$ & Idesia Arica \\
\hline
\end{tabular}


Paula Luíza Santos, Robério Anastácio Ferreira, Alexsandro

Guimarães de Aragão, Luise Andrade Amaral, Andreza Santos Oliveira, 2012.

Alexandre Marques da Silva, Daniela Sílvia de Oliveira Canuto, Mario Luiz Teixeira de Moraes, Salatiér Buzetti, 2012.

Ségio Alves de Sousa, Joedna Silva, Danilo Pereira Ramos, Taynar Coelho de Oliveira, Luis Antônio de Menezes Gonzaga, Rodrigo Ribeiro Fidelis, 2012.

Leila Paula Tonello, Joedna Silva, Danilo Pereira Ramos, Sérgio Alves Sousa, Rodrigo Ribeiro Fidelis, 2012.

Rodrigo Tenório de Vasconcelos, 2012.

Eichert, 2013.

Júlia Ferreira Brito, Karolline Sena Figuerêdo, Michelli, Medeiros Cabral Ribeiro, Antônio Carlos Martins dos Santos, Rubens Ribeiro da Silva, 2013.

Nádia Regina Lenhard, Vespasiano Borges de Paiva Neto, Silvana de Paula Quintão Scalon, Amauri Alves de Alvarenga, 2013.

Francisco V. da S. Sá, Marcos E. B. Brito, Alberto S. de Melo, Pedro Antônio Neto, Pedro D. Fernandes, Ilkelan B. Ferreira, 2013.

Elonha Rodrigues dos Santos, Adão Felipe dos Santos, Aristóteles Capone, Wembles Ribeiro dos Santos, Stefany Gregory Moura, Hélio Bandeira Barros, 2013.

Thiago Gledson Rios Terra,Tarcísio Castro Alves de Barros Leal,Aluízio Borém, Paulo Hideo Nakano Rangel, 2013.

Joice MariAssmann, IbanorAnghinoniaAmanda PosseltMartins, Sérgio Ely Valadão Gigante de AndradeCosta, DiegoCecagno, Filipe SelauCarlos, Paulo Cesar de FaccioCarvalho, 2014.

Luderlândio de A. Silva, Marcos E. B. Brito, Francisco V. da S. Sá, Rômulo C. L. Moreira, Walter dos S. Soares Filho, Pedro D.

Fernandes, 2014.

Marco Bruno Xavier Valadão, Ben Hur Marimon Junior, Paulo Sérgio Morandi, Simone Matias Reis, Bianca de Oliveira, Edmar Almeida de Oliveira e Beatriz Schwantes Marimon, 2014.

Rubens Cherubini Alves,Carolina Bremm, Cleist Luiz Ribeiro Nunes, Raquel Santiago Barro, Armindo Barth Neto, Radael Marinho Tres Schons, Luis Augusto Martins Caetano, Paulo César de Faccio Carvalho, 2015.

Petterson Baptista Luz, Thalita Neves Marostega, Raphael Egues Ranzani, Edileuson Galvão Aleixo, Deizimary Stella de Araújo, Guilherme Koch, Leonarda Grillo Nenes, 2015.

Adalberto Alves Pereira e Edivaldo Lopes Thomaz, 2015.

Daniele Rodrigues GomesMaristela, Machado AraujoUbirajara Rossi,
Estabelecimento de espécies florestais nativas por meio de semeadura direta para recuperação de áreas degradadas.

Avaliação das propriedades químicas em solo de cerrado sob reflorestamento ciliar.

Eficiência e resposta à aplicação de nitrogênio de genótipos de feijão comum cultivados em várzea tropical do Estado do Tocantins.

Eficiência do uso de fósforo em genótipos de arroz cultivados em solos de terras altas.

Enraizamento de estacas de Khaya senegalensis A. Juss. em diferentes concentrações de ácido indolbutírico.

Foliar Nutrient uptake - of myths and legends

Tratamentos pré-germinativos em sementes de Sclerolobium denudatum Vogel.

Crescimento de mudas de pau-ferro sob diferentes níveis de sombreamento.

Produção de mudas de mamoeiro irrigadas com água salina.

Journal of Biotechnology and

Biodiversity

Revista Árvore

Floresta

Revista Verde de

Agroecologia e

Desenvolvimento Sustentável

Repositório Institucional UNESP

Acta Horticulturae

Journal of Biotechnology and Biodiversity

Pesquisa Agropecuária Tropical

Revista Brasileira de Engenharia Agrícola e Ambiental

Dissimilaridade genética entre genótipos de soja cultivados em várzea irrigada no Journal of Biotechnology and Biodiversity período de entressafra.

Tolerância de linhagens de arroz de terras altas à seca.

Pesquisa Agropecuária Tropical

Soil carbon and nitrogen stocks and fractions in a long-term integrated croplivestock system under no-tillage in southern Brazil.

Mecanismos fisiológicos em híbridos de citros sob estresse salino em cultivo hidropônico.

Agriculture, ecosystems \& environment

Initial development and biomass partitioning of Physocalymma scaberrimum Pohl (Lythraceae) under different shading levels.

Suprimento de nitrogênio para culturas de verão pela aplicação antecipada em azevém pastejado por ovinos.

Revista Brasileira de Engenharia Agrícola e Ambiental

Scientia Forestalis

Costus cuttings development on indolebutyric acid concentrations.

Revista Brasileira de Ciência do Solo

Científica

Atributos químicos do solo em áreas sob diferentes sistemas de uso e manejo no município de Reserva-PR.

Biometria e germinação de Journal of Seed Science 


\begin{tabular}{|c|c|c|}
\hline NunesSuelen Carpenedo Aimi, 2015. & Balfourodendron riedelianum Eng. & \\
\hline $\begin{array}{l}\text { Paulo Affonso Fonseca Pires Neto, Victória Campos Monteiro Pires, } \\
\text { Cristiano Bueno Moraes, Lucicleia Mendes de Oliveira, Augustus } \\
\text { Caeser Franke Portella, João Nakagawa, } 2016 .\end{array}$ & $\begin{array}{l}\text { Physiological ripening of Anadenanthera } \\
\text { colubrina (Vellozo) Brenan seeds. }\end{array}$ & Journal of Seed Science \\
\hline Gustavo Mariano Rezende, 2016. & $\begin{array}{l}\text { Restauração florestal no sul da } \\
\text { Amazônia: métodos para romper } \\
\text { barreiras à regeneração natural. }\end{array}$ & Repositório UNB \\
\hline João Paulo Ascari \& Inês Roeder Nogueira Mendes, 2017. & $\begin{array}{l}\text { Desenvolvimento agronômico e } \\
\text { produtivo da soja sob diferentes doses de } \\
\text { gesso agrícola }\end{array}$ & Revista Agrogeoambiental \\
\hline $\begin{array}{l}\text { Janisson Batista de Jesus, Robério Anastácio Ferreira, Dráuzio } \\
\text { Correia Gama, João Horácio Almeida de Goes, } 2017 .\end{array}$ & $\begin{array}{l}\text { Estabelecimento de espécies florestais } \\
\text { nativas via semeadura direta no rio } \\
\text { Piauitinga-Sergipe. }\end{array}$ & Floresta e Ambiente \\
\hline $\begin{array}{l}\text { Leila Cristiane-Delmadi, Cristiane de Pieri, Alex Sander-Porcena, } \\
\text { Edson Luiz-Furtado, } 2017 .\end{array}$ & $\begin{array}{l}\text { Diagramatic scale for quantification of } \\
\text { rust severity in teak leaves. }\end{array}$ & $\begin{array}{l}\text { Revista mexicana de } \\
\text { fitopatología }\end{array}$ \\
\hline $\begin{array}{l}\text { Jania Claudia Camilo dos Santos, Dayane Mércia Ribeiro Silva, } \\
\text { Renato Nunes Costa, Carlos Humberto da Silva, Wilton da Silva } \\
\text { Santos, Flávia de Barros Prado Moura, José Vieira Silva, } 2017 .\end{array}$ & $\begin{array}{l}\text { Aspectos biométricos e morfológicos de } \\
\text { frutos e sementes de Schinopsis } \\
\text { brasiliensis. }\end{array}$ & Nativa \\
\hline $\begin{array}{l}\text { Deyse Cristina Oliveira da Silva, Sandra Catia Pereira Uchôa, José } \\
\text { Maria Arcanjo Alves, Leandro Torres de Souza, Cineone Nascimento } \\
\text { da Silva, Glauber Ferreira Barreto, Laís de Brito Carvalho e Arthur } \\
\text { José Evangelista dos Anjos, } 2020 .\end{array}$ & $\begin{array}{l}\text { Adubação foliar na suplementação } \\
\text { nutricional de cultivares de abacaxizeiro } \\
\text { micropropagadas. }\end{array}$ & Investigación Agraria \\
\hline $\begin{array}{l}\text { Pedro Paulo Gomes de Oliveira, Hugo Armando Barúa Acosta, } \\
\text { Luciano de Souza Maria e Marco Antônio Camillo de Carvalho, } 2020 .\end{array}$ & $\begin{array}{l}\text { Influência da época e do número de } \\
\text { desrama sobre o desenvolvimento inicial } \\
\text { de Tectona grandis } 1 . \text { F. no sistema } \\
\text { silvipastoril. }\end{array}$ & Investigación Agraria \\
\hline $\begin{array}{l}\text { Deyse Cristina Oliveira da Silva, Sandra Catia Pereira Uchôa, José } \\
\text { Maria Arcanjo Alves, Valdinar Ferreira Melo, Cineone Nascimento da } \\
\text { Silva, Glauber Ferreira Barreto, } 2020 .\end{array}$ & $\begin{array}{l}\text { Initial Growth of Seedlings of Diferent } \\
\text { Pineapple Cultivars in Boa Vista, RR. }\end{array}$ & $\begin{array}{l}\text { Ensaios e Ciência C } \\
\text { Biológicas Agrárias e da } \\
\text { Saúde }\end{array}$ \\
\hline
\end{tabular}

Fonte: Autores (2021).

Em relação aos temas abordados, pode-se verificar que $23(56,0 \%)$ dos artigos selecionados se enquadram, de acordo com a tabela de classificação da CAPES, na área de Agronomia, sendo que desses vinte três estudos $3(7,3 \%)$ pertencem a subárea da Ciência do Solo; 1 (2,4\%) a Fitossanidade; 17 (41,4\%) a Fitotecnia; 2 (4,8\%) a Floricultura, Parques e Jardins. Ademais, percebeu-se que 18 (43,9\%) dos artigos selecionados pertencem a área de Recursos florestais e engenharia florestal, desse montante $12(29,2 \%)$ pertencem a subárea da Silvicultura; 2 (4,8\%) a Manejo Florestal; 1 (2,4\%) a Tecnologia e Utilização de Produtos Florestais; e 3 (7,3\%) Conservação da Natureza. Nenhum dos estudos selecionados são classificados em Engenharia agrícola.

\section{Discussão}

\section{Aplicação do delineamento em blocos casualizados na ciências agrárias}

Para Silva, Morais e Buzetti (2011) e Silva et al. (2012) em seus trabalhos avaliaram as propriedades químicas de solo sob reflorestamento ciliar de plantio em área de Cerrado com delineamento em blocos casualizados em esquema hierárquico. Os resultados mostram que as propriedades químicas do solo de reflorestamento ciliar são semelhantes às do solo não perturbado desta área. As espécies plantadas no reflorestamento ciliar ajudam a depositar matéria orgânica suficiente para a ciclagem de nutrientes e mantêm as propriedades químicas do solo em boas condições para o estabelecimento das matas ciliares, Pereira e Thomaz (2015) observaram resultados análogos. 
No trabalho de Oliveira et al. (2020) e Eichertn (2012) teve como foco identificar os efeitos de composições de adubo foliar no crescimento e produção de cinco cultivares de abacaxizeiro micropropagadas, foi utilizado o delineamento experimental em blocos casualizados, em parcelas subdivididas com três fatores e quatro repetições, obtendo os resultados e os submetendo à análise de variância pelo teste F, empregando também o teste de Scott-Knott para as comparações entre cultivares e adubação, e de regressão para as épocas, empregando-se o programa SISVAR. Os valores obtidos confirmaram que IAC Fantástico sobressaiu na maioria dos caracteres avaliados menos com a adubação submetida e também apresentou características vegetativas e produtivas superiores e podendo ser uma opção viável de cultivo no estado de Roraima. No trabalho de Silva et al. (2020), obteve resultados próximos aos dos autores mencionados anteriormente.

De acordo com Oliveira et al. (2020), Vieira et al. (2010) e Cristiane-Delmad et al. (2018), em seus trabalhos com vertentes semelhantes avaliaram o número e a época de desrama e sua influência sobre o desenvolvimento em altura, diâmetro e a qualidade do fuste de um povoamento em espécies distintas, o delineamento experimental utilizado foi em blocos casualizados, com quatro tratamentos (desrama três vezes ao ano, desrama somente em fevereiro, desrama somente em agosto e desrama somente em novembro) e cinco repetições com oito árvores por parcela totalizando 160 árvores. Analisou o DAP $(1,30 \mathrm{~m})$, altura total, incremento corrente anual em altura e diâmetro e a qualidade do fuste. A aplicação do tratamento (desrama realizada no mês de fevereiro) resultou no desenvolvimento inicial de povoamento, sendo vantajoso.

Dias et al. (2011), Vasconcelos (2012) e Luz et al. (2015), a fim de avaliar o efeito de diferentes concentrações e meios de diluição de ácido indolbutírico (AIB) no enraizamento de estacas semilenhosas de cerejeira ornamental Prunus serrulata Lind L. Utilizaram o delineamento blocos casualizados em fatorial 3x2 com 4 repetições e 5 plantas por parcela. Não observaram influência de doses nem de veículo de aplicação do AIB nas características de porcentagem de estacas com gemas, número de brotações e porcentagem de estacas brotadas. Em comparação com outros tratamentos, a concentração de 1.000 mg.L-1 aplicada com líquido apresentou maior porcentagem de enraizamento, número de raízes e comprimento da raiz mais longa, porém ainda é considerada baixa e necessita de estudos mais aprofundados.

Ascari e Mendes (2017) e Caires et al. (2011), verificaram a influência de diferentes dosagens de gesso agrícola no desenvolvimento vegetativo e reprodutivo da cultura da soja. O delineamento experimental foi em blocos casualizados (DBC) com cinco tratamentos, sendo 0, 1, 2, 3 e 4 t ha-1 de gesso agrícola, com quatro repetições. No resultados foram observados que as variáveis altura de planta, altura da primeira vagem, número de nós, número de vagens por planta, massa de 100 grãos e produtividade foram influenciadas de forma significativa pelas doses de gesso agrícola. Confirmando que a dose de $2 \mathrm{t}$ ha- 1 de gesso agrícola apresentou o melhor efeito no desenvolvimento vegetativo e reprodutivo da soja.

Medeiros et al. (2011), verificaram o efeito de substratos na atratividade de moscas-das-frutas, nas culturas da goiabeira e da mangueira. O delineamento experimental utilizado para ambas as espécies avaliadas foi o de blocos casualizados (DBC), o que diferiram foram a quantidade de tratamentos e repetições, a da goiabeira com cinco tratamentos e quatro repetições. Para as plantas de mangueira, foram aplicados três tratamentos e quatro repetições. Concluíram que: os tratamentos foram eficientes para realizar o monitoramento das moscas-das-frutas nos pomares de goiabeira e mangueira; foram observados a presença das seguintes espécies de moscas-das-frutas: Ceratitis capitata, Anastrepha fraterculus, A. sororcula, A. zenildae e A. obliqua.

Santos et al. (2012), avaliaram os efeitos da salinidade da água de irrigação sobre a condutividade elétrica do extrato da pasta saturada do solo (CEes) e as alterações dos parâmetros fisiológicos e de produtividade da cultura do amendoim, utilizaram também o delineamento em blocos casualizados, com seis tratamentos e quatro repetições. Os resultados mostraram que a CEes diminuiu significativamente com o aumento das frações de lixiviação, o decréscimo do potencial osmótico foliar na planta de amendoim implicou na redução da produtividade e o teor de clorofila e a massa de matéria seca total reduziram 
significativamente com o aumento da CEes. Isso foi verificado em outros estudos quando as plantas são submetidas ao estresse salino (Medeiros et al., 2011; Maciel et al., 2012).

Fernandes et al. (2011), Sá et al. (2013), Silva et al. (2014), estudaram a sensibilidade à salinidade durante a fase de formação de porta-enxertos compreendendo variedades e híbridos selecionados, utilizando delineamento em blocos casualizados, com cinco repetições em esquema fatorial e quatro plantas úteis por parcela, durante o período de 150 dias. Avaliaram-se variáveis de crescimento e fisiológicas. A redução mais expressiva foi observada na fitomassa seca total da parte aérea com aumento unitário da CEa. Observou-se redução linear no crescimento com aumento da salinidade nos híbridos LVK x LVA-009 e TSK x TRENG-256. Dentre os genótipos avaliados, o limoeiro 'Volkameriano' foi o menos sensível ao estresse salino.

Sandini et al (2011), Alves et al. (2015) e Assmann et al. (2014), avaliaram o efeito residual da aplicação de N na pastagem, com e sem pastejo, sobre a cultura do milho. O experimento foi realizado em delineamento de blocos casualizados com parcelas subsubdivididas. A produtividade de grãos, assim como o número de fileiras e grãos por espiga não foram afetados pelo pastejo. Houve resposta quadrática na produtividade de grãos em função do aumento das doses de N-TI e N-TV.

Dutra et al. (2012), avaliou a influência de diferentes níveis de sombreamento e tipos de substratos no desenvolvimento inicial de mudas de copaíba. O experimento foi conduzido no delineamento em blocos casualizados no esquema fatorial $5 \times 4$. Os resultados demonstram que as mudas de copaíba necessitam de sombra em sua fase inicial de desenvolvimento, sendo o nível de 50\% de sombreamento uma alternativa viável para produção de suas mudas. Resultados semelhantes foram observados nos trabalhos de Oliveira e Perez (2012), Lenhard et al. (2013) e Valadão et al. (2014).

Santos et al. (2012), Jesus et al. (2017) e Santos (2018), avaliaram a utilização da semeadura direta e a influência de um protetor físico sobre o estabelecimento de espécies florestais nativas em uma área com pastagem e outra com agricultura e utilização o delineamento em blocos casualizados, em esquema fatorial. As avaliações feitas em campo foram: emergência, sobrevivência e desenvolvimento inicial das mudas das espécies (altura e diâmetro). Com relação à emergência e sobrevivência de plântulas, houve diferença significativa entre os dois ambientes, com melhores resultados no subsistema agrícola. A semeadura direta foi viável na recuperação de áreas degradadas com espécies florestais nativas, nos ambientes estudados, resultados obtidos também nos trabalhos de Gomes et al. (2016), Pires Neto (2016) e Rezende (2016).

Brito et al. (2013) analisaram diferentes métodos de superação de dormência em sementes (AVELINO, 2012) de Sclerolobium denudatum Vogel aproveitadas para a recuperação de áreas degradadas, para os ensaios utilizaram o delineamento em blocos casualizados com quatro repetições, sendo esses: testemunha, imersão em ácido sulfúrico, escarificação mecânica com lixa grana e de imersão das sementes em água, imersão das sementes em água e imersão em esterco bovino fresco. A maior porcentagem de germinação foi obtida no tratamento onde as sementes foram imersas em ácido sulfúrico 70\% por 25 min. Imersão em esterco bovino fresco, imersão em água e escarificação mecânica com lixa grana 100 seguida por imersão em água reduzem a taxa de germinação das sementes de Sclerolobium denudatum Vogel.

Santos et al. (2013), utilizou o delineamento experimental em blocos casualizados com quatro blocos para avaliar a diversidade genética entre 18 genótipos de soja por meio de técnicas multivariadas baseadas em 17 características fisiológicas e agronômicas. Sousa et al. (2012), Tonello et al. (2012) e Terra et al. (2013), avaliaram o efeito da adubação nitrogenada em genótipos de feijão comum cultivados em solos de várzea irrigada. $\mathrm{O}$ estudo foi conduzido em dois experimentos (alto e baixo nitrogênio) com delineamento em blocos casualizados. Em todas as análises os genótipos estudados apresentaram aptidão para colheita mecanizada e também resposta positiva à adubação nitrogenada.

Teixeira et al. (2010), avaliou características agronômicas e qualidades fisiológicas de sementes de diferentes cultivares de feijão-caupi na condições edafoclimáticas do cerrado goiano. Foi empregado o delineamento em blocos 
casualizados com quatro repetições. O efeito dos tratamentos foram significativos para todos os caracteres avaliados nas espécies de feijão-caupi estudados.

\section{Considerações Finais}

Os resultados deste estudo demostram que a técnica da estatística experimental "Delineamento em Blocos Casualizados (DBC)" aplicado em pesquisas nas áreas da Ciências Agrárias tem contribuído para o avanço dos diferentes campos, possibilitando uma melhor tabulação dos dados adquiridos, e facilitando na análise das unidades experimentais. Além do mais, observou-se que na grande área das Ciências Agrárias os ramos de estudo da Agronomia, Recursos florestais e engenharia florestal são os que desenvolvem mais pesquisas com o DBC, com ênfase nas subáreas de Fitotecnia e Silvicultura.

Portanto, fica evidente a importância desse tipo de delineamento nos campos de estudo que comparam mais de uma variável, e possuem abordagens totalmente dependentes do meio, tais como: propriedades químicas do solo, adubo foliar para produção de frutíferas, manejo florestal, produção de mudas, produção de culturas agrícolas, irrigação, agroflorestal, superação de dormência de sementes, genética de culturas, dentre outras.

Este estudo descreveu relações entre os construtos práticos do DBC nas pesquisas em Ciências Agrárias, a partir de uma amostra das áreas de Engenharia agrícola (na qual não foi encontrado nenhum artigo dentro dos critérios de inclusão), Agronomia, Recursos florestais e engenharia florestal. Consequentemente, não é possível estender os resultados obtidos para as demais áreas que compõe na grande área de Ciências Agrárias.

Assim, recomenda-se para pesquisas futuras uma investigação que aborda as demais áreas incluídas na grande área de Ciências Agrárias, objetivando identificar estudos que utilizam o DBC, para gerar evidencias empíricas da abrangência desse método estatístico e suas contribuições no desenvolvimento de outros estudos.

\section{Referências}

Alves, G. P. (2019) Planejamento de experimentos. Centro Universitário Leonardo Da Vinci, Indaial: UNIASSELVI, 212 p.

Alves, R. C. et al. (2015) Suprimento de nitrogênio para culturas de verão pela aplicação antecipada em azevém pastejado por ovinos. Revista Brasileira de Ciência do Solo, 39 (5), 1406-1415. https://doi.org/10.1590/01000683rbcs20151048

Ascari, J., \& Mendes, I. (2017). Desenvolvimento agronômico e produtivo da soja sob diferentes doses de gesso agrícola. Revista Agrogeoambiental, 9 (4). http://dx.doi.org/10.18406/2316-1817v9n420171014

Assmann, J. M. et al. (2014). Soil carbon and nitrogen stocks and fractions in a long-term integrated crop-livestock system under no-tillage in southern Brazil. Agriculture, ecosystems \& environment, 190, 52-59. https://doi.org/10.1016/j.agee.2013.12.003

Avelino, J. I., Lima, J. S. S., Ribeiro, M. C. C., Chaves, A. P. \& Rodrigues, G. S. O. (2012). Métodos de quebra de dormência em sementes de jucá (Caesalpinia ferrea Mart. ex Tul. var. Ferrea). Revista Verde de Agroecologia e Desenvolvimento Sustentável, 7 (1), $102-106$.

Brito, J., Figuerêdo, K. S., Ribeiro, M. M. C., dos Santos, A. C. M., \& da Silva, R. R. (2013). Tratamentos pré-germinativos em sementes de Sclerolobium denudatum Vogel. Journal of Biotechnology and Biodiversity, 4 (4), 365-370. https://doi.org/10.20873/jbb.uft.cemaf.v4n4.brito.

Caires, E. F., Maschietto, E. H. G., Garbuio, F. J., Churka, S. \& Joris, H. A. (2011). Surface application of gypsum in low acidic Oxisol under no-till cropping system. Scientia Agricola, Piracicaba, 68 (2), 209-216. https://doi.org/10.1590/S0103-90162011000200011

Cargnelutti Filho, A. (2009). Experimentação agrícola e florestal. Santa Maria: UFSM / CCR / Departamento de Fitotecnia, 204 p.

Coordenação de Aperfeiçoamento de Pessoal de Nível Superior. CAPES. (2021.) Tabela das Áreas de Conhecimento. https://www.gov.br/capes/pt-br/acessoa-informacao/acoes-e-programas/avaliacao/instrumentos/documentos-de-apoio-1/tabela-de-areas-de-conhecimento-avaliacao

Cristiane-Delmadi, L., Pieri, C., Sander-Porcena, A. \& Luiz-Furtado, E. (2018). Diagramatic scale for quantification of rust severity in teak leaves. Revista mexicana de fitopatología, 36 (2), 331-341. https://doi.org/10.18781/r.mex.fit.1708-5

Costa, J. R. (2003) Técnicas Experimentais aplicadas às Ciências Agrárias. EMBRAPA- Agrobiologia, Seropédica - RJ, p. 1-54.

Dias, M. M., Chalfun, N. N. J., Coelho, S. J. \& Santos, V. A. (2011). Meios de diluição e concentrações de ácido indolbutírico no enraizamento de estacas de cerejeira ornamental. Tecnol. \& Ciên. Agropec, 5 (4), 39-43.

Dutra, T. R. et al. (2012). Desenvolvimento inicial de mudas de copaíba sob diferentes níveis de sombreamento e substratos. Revista Ciência Agronômica. 43 (2), 321-329. https://doi.org/10.1590/S1806-66902012000200015 
Eichert, T. (2013). Foliar Nutrient uptake - of myths and legends. Acta Horticultura 984, 69-75. https://doi.org/10.17660/ActaHortic.2013.984.5

Fernandes, P. D. et al. (2011). Crescimento de híbridos e variedades porta-enxerto de citros sob salinidade. Acta Scientiarum. Agronomy, 33 (2), $259-267$. https://doi.org/ 10.4025/actasciagron.v33i2.5582

Gomes, D. R. et al. (2016) Biometria e germinação de Balfourodendron riedelianum Eng. Journal of Seed Science, 38 (3), 187-194. https://doi.org/10.1590/2317-1545v38n3159311

Grupo Anima Educação. (2014). Manual Revisão Bibliográfica Sistemática Integrativa: a pesquisa baseada em evidências. Belo Horizonte: Grupo Anima Educação.

Jesus, J. B. et al. (2017). Estabelecimento de espécies florestais nativas via semeadura direta no rio Piauitinga-Sergipe. Floresta e Ambiente, 24. https://doi.org/10.1590/2179-8087.028815

Lenhard, N. R. et al. (2013). Crescimento de mudas de pau-ferro sob diferentes níveis de sombreamento. Pesquisa Agropecuária Tropical, 43 (2), 178-186. https://doi.org/10.1590/S1983-40632013000200012

Luz, P. B. et al. (2015). Costus cuttings development on indolebutyric acid concentrations. Científica, 43 (4), 336-340. http://dx.doi.org/10.15361/1984$5529.2015 \mathrm{v} 43 \mathrm{n} 4 \mathrm{p} 336-340$

Kronka, S. N. (2018). Estatística experimental. FUNEP, 54 p.

Maciel, M. P., Soares, T. M., Gheyi, H. R., Rezende, E. P. L., \& Oliveira, G. X. S. (2012). Produção de girassol ornamental com uso de águas salobras em sistema hidropônico NFT. Revista Brasileira de Engenharia Agrícola e Ambiental. 16 (2), 165-172. https://doi.org/10.1590/S1415-43662012000200006

Medeiros, D. C., Medeiros, J. F., Dias, P. M. S., Barbosa, M. A. G. \& Lima, K. S. (2011). Produção de melão Pele de Sapo híbrido 'Medellín' cultivado com diferentes salinidades da água. Revista Ciência Agronômica, 42 (3), 628-634. https://doi.org/10.1590/S1806-66902011000300008

Medeiros, J. G. F., Malta, A. O., Costa, N. P., Araújo, R. C. \& Araújo, E. L. (2011). Substâncias Atrativas no Monitoramento de Moscas-dasfrutas em Goiabeiras e Mangueiras no Município de Bananeiras-PB. Revista Verde de Agroecologia e Desenvolvimento Sustentável, 6 (5), 213-219.

Oliveira, A. K. M. \& Perez, S. C. J. G. A. (2012). Crescimento inicial de Tabebuia aurea sob três intensidades luminosas. Ciência Florestal, 22 (2), $263-273$. https://doi.org/10.5902/198050985733

Oliveira da Silva, D. C. et al. (2020). Adubação foliar na suplementação nutricional de cultivares de abacaxizeiro micropropagadas. Investigación Agraria, 22(1), 22-29. http://dx.doi.org/10.18004/investig.agrar.2020.junio.22-29

Oliveira, G. P. P., Acosta, B. H. A., Maria, S. L., \& Carvalho, C. M. A. (2020). Influência da época e do número de desrama sobre o desenvolvimento inicial de Tectona grandis 1. F. no sistema silvipastoril. Investigación Agraria, 22(1), 39-45. http://dx.doi.org/10.18004/investig.agrar.2020.junio.39-45

PadovanI, C. R. (2014). Delineamento de experimentos. Cultura Acadêmica, 128 p.

Pereira, A. A. \& Thomaz, E. L. (2015) Atributos químicos do solo em áreas sob diferentes sistemas de uso e manejo no município de Reserva-PR. Caminhos de geografia, 16 (55), 186-194.

Pimentel-Gomes, F. (2009). Curso de Estatística Experimental. Piracicaba: FEALQ, 451 p.

Pires Neto, P. A. F. et al. (2016). Physiological ripening of Anadenanthera colubrina (Vellozo) Brenan seeds. Journal of Seed Science, 38 (2), 155-161.

Rezende, G. M. (2016). Restauração florestal no sul da Amazônia: métodos para romper barreiras à regeneração natural. Repositório UNB.

Sá, F. V. S. et al. (2013). Produção de mudas de mamoeiro irrigadas com água salina. Revista Brasileira de Engenharia Agrícola e Ambiental, 17 (10), 10471054.

Sandini, I. E. et al. (2011). Efeito residual do nitrogênio na cultura do milho no sistema de produção integração lavoura-pecuária. Ciência Rural, 41 (8), 13151322. https://doi.org/10.1590/S0103-84782011005000099

Santos, D. B. S, Ferreira, P. A., Oliveira, F. G., Batista, R. O., Costa, A. C. \& Cano, M. A. O. (2012). Produção e parâmetros fisiológicos do amendoim em função do estresse salino. Idesia (Arica), 30 (2), 69-74. http://dx.doi.org/10.4067/S0718-34292012000200009

Santos, E. R., Dos Santos, A. F., Capone, A., Dos Santos, W. R., Moura, S. G., \& Barros, H. B. (2013). Dissimilaridade genética entre genótipos de soja cultivados em várzea irrigada no período de entressafra. Journal of Biotechnology and Biodiversity, 4 (3), $222-231$. https://doi.org/10.20873/jbb.uft.cemaf.v4n3.santos

Santos, P. L. et al. (2012). Estabelecimento de espécies florestais nativas por meio de semeadura direta para recuperação de áreas degradadas. Revista Árvore, 36 (2), 237-245. https://doi.org/10.1590/S0100-67622012000200005

Santos, J. C. C. et al. (2018). Aspectos biométricos e morfológicos de frutos e sementes de Schinopsis brasiliensis. Nativa, v. 6 (3), $219-224$. https://doi.org/10.31413/nativa.v6i3.4709

Silva, A. M., Moraes, M. L. T. \& Buzetti, S. (2011). Propriedades químicas de solo sob reflorestamento ciliar após 20 anos de plantio em área de cerrado. Revista Árvore, 35 (1), 97-106. https://doi.org/10.1590/S0100-67622011000100012

Silva, Alexandre Marques et al. (2012). Avaliação das propriedades químicas em solo de cerrado sob reflorestamento ciliar. Floresta, 42 (1), $49-58$. 
Research, Society and Development, v. 10, n. 8, e53510817616, 2021

(CC BY 4.0) | ISSN 2525-3409 | DOI: http://dx.doi.org/10.33448/rsd-v10i8.17616

Silva, Deyse Cristina Oliveira et al. (2020) Initial Growth of Seedlings of Diferent Pineapple Cultivars in Boa Vista, RR. Ensaios e Ciência C Biológicas Agrárias e da Saúde, 24 (1), 41-46. https://doi.org/10.17921/1415-6938.2020v24n1p41-46

Silva, L. A. et al. (2014). Mecanismos fisiológicos em híbridos de citros sob estresse salino em cultivo hidropônico. Revista Brasileira de Engenharia Agrícola e Ambiental, 18, 1-7. https://doi.org/10.1590/1807-1929/agriambi.v18nsuppS1-S7

Souza, M. T., Silva, M. D. \& Carvalho, R. (2010) Revisão integrativa: o que é e como fazer. Einstein, São Paulo, 8 (1), 102-6. http://www.astresmetodologias.com/material/O_que_e_RIL.pdf

Sousa, S. A., Silva, J., Ramos, D. P., De Oliveira, T. C., Gonzaga, L. A. M., \& Fidelis, R. R. (2012). Eficiência e resposta à aplicação de nitrogênio de genótipos de feijão comum cultivados em várzea tropical do Estado do Tocantins. Journal of Biotechnology and Biodiversity, 3 (3), 31-37. https://doi.org/10.20873/jbb.uft.cemaf.v3n3.sousa

Terra, T. G. R. et al. (2013). Tolerância de linhagens de arroz de terras altas à seca. Pesquisa Agropecuária Tropical, 43 (2), $201-208$. https://doi.org/10.1590/S1983-40632013000200013

Teixeira, I. R. et al. (2010). Desempenho agronômico e qualidade de sementes de cultivares de feijão-caupi na região do cerrado. Revista Ciência Agronômica, 41 (2), 300-307. https://doi.org/10.1590/S1806-66902010000200019

Tonello, L. P. et al. (2012). Eficiência do uso de fósforo em genótipos de arroz cultivados em solos de terras altas. Revista Verde de Agroecologia e Desenvolvimento Sustentável, 7 (2), 25-32.

Valadão, M. B. X. et al. (2014). Initial development and biomass partitioning of Physocalymma scaberrimum Pohl (Lythraceae) under different shading levels. Scientia Forestalis, 42 (101), 129-139.

Vasconcelos, R. T. (2012). Enraizamento de estacas de Khaya senegalensis A. Juss. em diferentes concentrações de ácido indolbutírico. Repositório Institucional UNESP.

Vieira, A. H. et al. (2010). Influência da desrama artificial sobre o crescimento da teca (Tectona grandis) no Estado de Rondônia. Embrapa Rondônia. Circular técnica. 